\title{
APPROXIMATIVE CHARACTERISTICS OF THE NIKOL'SKII-BESOV-TYPE CLASSES OF PERIODIC FUNCTIONS IN THE SPACE $B_{\infty, 1}$
}

\author{
FedunYK-YAREMChuK O.V., Hembars'Kyi M.V., Hembars'Ka S.B.
}

\begin{abstract}
We obtained the exact order estimates of the orthowidths and similar to them approximative characteristics of the Nikol'skii-Besov-type classes $B_{p, \theta}^{\Omega}$ of periodic functions of one and several variables in the space $B_{\infty, 1}$. We observe, that in the multivariate case $(d \geq 2)$ the orders of orthowidths of the considered functional classes are realized by their approximations by step hyperbolic Fourier sums that contain the necessary number of harmonics. In the univariate case, an optimal in the sense of order estimates for orthowidths of the corresponding functional classes there are the ordinary partial sums of their Fourier series. Besides, we note that in the univariate case the estimates of the considered approximative characteristics do not depend on the parameter $\theta$. In addition, it is established that the norms of linear operators that realize the order of the best approximation of the classes $B_{p, \theta}^{\Omega}$ in the space $B_{\infty, 1}$ in the multivariate case are unbounded.

Key words and phrases: Nikol'skii-Besov-type class, orthowidth, best approximation.
\end{abstract}

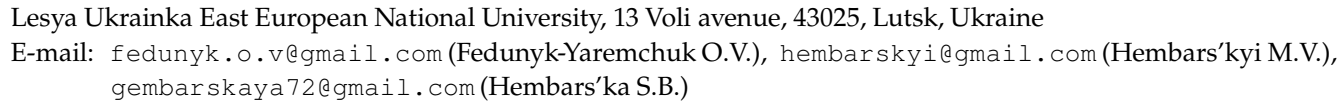

\section{INTRODUCTION}

In this paper, we continue to study the approximative characteristics of the classes $B_{p, \theta}^{\Omega}$ of periodic functions of several variables and classes $B_{p, \theta}^{\omega}$ of one variable in the space $B_{\infty, 1}$, which norm is stronger than the norm in $L_{\infty}$. We recall that some approximative characteristics of functional classes in the space $B_{\infty, 1}$ were studied in $[7,14,15,27,28]$. As noted in these papers, the motivation to study the approximation characteristics (best approximation, widths, best $M$-term approximation etc.) of classes $B_{p, \theta}^{r}$ and $B_{p, \theta}^{\Omega}$ in the space $B_{\infty, 1}$ was the fact that the questions of their orders, especially in the multidimensional case, in the space $L_{\infty}$ still remain open (see also [10]).

The paper consists of two parts. In the first part, we obtain exact order estimates of orthowidths of classes $B_{p, \theta}^{\Omega}$ and $B_{p, \theta}^{\omega}$ in the space $B_{\infty, 1}$ and to them similar approximative characteristics as well. Analyzing the results obtained in this part of the paper, we pay attention to the following facts: in the one-dimensional case, estimates of the considered approximation characteristics do not depend on the parameter $\theta$; comparing the results of Theorems $4,6,8$ with estimates of the corresponding quantities in the space $L_{\infty}$, we see that they are identical in order. As for the multidimensional case, comparing the results of Theorems 4, 5, 7 with the corresponding estimates in the space $L_{\infty}$, we see that they also coincide, but instead depend on the parameter $\theta$. This is, in particular, a feature of the multidimensional case, compared 
with the one-dimensional case. We also observe, that in the multivariate case $(d \geq 2)$ the orders of orthowidths of the considered functional classes are realized by their approximations by step hyperbolic Fourier sums that contain the necessary number of harmonics. In the univariate case, an optimal in the sense of order estimates for orthowidths of the corresponding functional classes are the ordinary partial sums of their Fourier series.

The second part of the paper is devoted to the study of norms of linear operators that realize the orders of the best approximations of the above mentioned classes in the space $B_{\infty, 1}$. It was found that in the multidimensional case $(d \geq 2)$, such operators have unbounded norms.

\section{NOTATIONS, DEFINITIONS AND AUXILIARY STATEMENTS}

Let $\mathbb{R}^{d}$ denotes $d$-dimensional space with elements $x=\left(x_{1}, \ldots, x_{d}\right)$, and let

$$
(x, y)=x_{1} y_{1}+\ldots+x_{d} y_{d}
$$

be a scalar product of elements $x, y \in \mathbb{R}^{d}$. By $L_{p}\left(\pi_{d}\right), \pi_{d}=\prod_{j=1}^{d}[0 ; 2 \pi)$, we denote the space of functions $f(x)$ which are $2 \pi$-periodic in each variable and such that

$$
\|f\|_{p}=\left((2 \pi)^{-d} \int_{\pi_{d}}|f(x)|^{p} d x\right)^{1 / p}<\infty, \quad 1 \leq p<\infty, \quad\|f\|_{\infty}=\operatorname{esssup}_{x \in \pi_{d}}|f(x)|<\infty .
$$

Thus, we assume that for $f \in L_{p}\left(\pi_{d}\right)$ the condition

$$
\int_{0}^{2 \pi} f(x) d x_{j}=0, j=\overline{1, d}
$$

is satisfied. We denote the set of such functions by $L_{p}^{0}\left(\pi_{d}\right)$. Sometimes instead of $L_{p}\left(\pi_{d}\right)$ and $L_{p}^{0}\left(\pi_{d}\right)$, we use the simpler notations $L_{p}$ and $L_{p}^{0}$, respectively.

We denote the $l$ th difference of a function $f \in L_{p}^{0}, 1 \leq p \leq \infty$, with a step $h_{j}$ in the variable $x_{j}$ by the formula

$$
\Delta_{h_{j}}^{l} f(x)=\sum_{n=0}^{l}(-1)^{l-n} C_{l}^{n} f\left(x_{1}, \ldots, x_{j-1}, x_{j}+n h_{j}, x_{j+1}, \ldots, x_{d}\right)
$$

For $f \in L_{p}^{0}, 1 \leq p \leq \infty, h=\left(h_{1}, \ldots, h_{d}\right)$ and $t \in \mathbb{R}_{+}^{d}$ we introduce a mixed $l$ th difference

$$
\Delta_{h}^{l} f(x)=\Delta_{h_{1}}^{l} \ldots \Delta_{h_{d}}^{l} f(x)=\Delta_{h_{d}}^{l}\left(\ldots\left(\Delta_{h_{1}}^{l} f(x)\right)\right)
$$

and we denote the mixed modulus of continuity of order $l$ by

$$
\Omega_{l}(f, t)_{p}=\sup _{\substack{\left|h_{j}\right| \leq t_{j} \\ j=1, d}}\left\|\Delta_{h}^{l} f(\cdot)\right\|_{p}
$$


Let $\Omega(t)=\Omega\left(t_{1}, \ldots, t_{d}\right)$ be a given function of the type of mixed modulus of continuity of the order $l$. This means that the function $\Omega$ satisfies the following conditions:

1) $\Omega(t)>0, t_{j}>0, j=\overline{1, d}$, and $\Omega(t)=0$, if $\prod_{j=1}^{d} t_{j}=0$;

2) $\Omega(t)$ is nondecreasing in each variable;

3) $\Omega\left(m_{1} t_{1}, \ldots, m_{d} t_{d}\right) \leq\left(\prod_{j=1}^{d} m_{j}\right)^{l} \Omega(t), m_{j} \in \mathbb{N}, j=\overline{1, d}$;

4) $\Omega(t)$ is continuous for $t_{j} \geq 0, j=\overline{1, d}$.

Following S.N. Bernstein [8], we call the function of one variable $\varphi(\tau)$ almost increasing on $[a, b]$, if there exists a constant $C_{1}>0$, which does not depend on $\tau_{1}, \tau_{2}$, such that

$$
\varphi\left(\tau_{1}\right) \leq C_{1} \varphi\left(\tau_{2}\right), \quad a \leq \tau_{1} \leq \tau_{2} \leq b
$$

and almost decreasing on $[a, b]$, if there exists a constant $C_{2}>0$, which does not depend on $\tau_{1}, \tau_{2}$, such that

$$
\varphi\left(\tau_{1}\right) \geq C_{2} \varphi\left(\tau_{2}\right), \quad a \leq \tau_{1} \leq \tau_{2} \leq b .
$$

We assume that the function $\Omega(t), t \in \mathbb{R}_{+}^{d}$, satisfies also the conditions $\left(S^{\alpha}\right)$ and $\left(S_{l}\right)$, which are called the Bari-Stechkin conditions $[5,31]$. This means the following.

A function of one variable $\varphi(\tau) \geq 0, \tau \in[0,1]$, satisfies the condition $\left(S^{\alpha}\right)$ if $\varphi(\tau) / \tau^{\alpha}$ almost increases for some $\alpha>0$.

A function $\varphi(\tau) \geq 0, \tau \in[0,1]$, satisfies the condition $\left(S_{l}\right)$ if $\varphi(\tau) / \tau^{\gamma}$ almost decreases for some $0<\gamma<l, l \in \mathbb{N}$.

In the case of $d>1$ we say that $\Omega(t), t \in \mathbb{R}_{+}^{d}$, satisfies these conditions if $\Omega(t)$ satisfies these conditions in each variable $t_{j}$ for fixed $t_{i}, i \neq j$.

We now define the functional classes $B_{p, \theta}^{\Omega}$, which were considered in the paper [37] by S. Yongshen, W. Heping.

Let $1 \leq p, \theta \leq \infty$ and let $\Omega(t)$ be a given function of the type of mixed modulus of continuity of the order $l$, which satisfies conditions 1$)-4),\left(S^{\alpha}\right)$ and $\left(S_{l}\right)$. Then the classes $B_{p, \theta}^{\Omega}$ are defined as follows

$$
B_{p, \theta}^{\Omega}=\left\{f \in L_{p}^{0}\left(\pi_{d}\right):\|f\|_{B_{p, \theta}^{\Omega}} \leq 1\right\}
$$

where

$$
\begin{gathered}
\|f\|_{B_{p, \theta}^{\Omega}}=\left\{\int_{\pi_{d}}\left(\frac{\Omega_{l}(f, t)_{p}}{\Omega(t)}\right)^{\theta} \prod_{j=1}^{d} \frac{d t_{j}}{t_{j}}\right\}^{1 / \theta}, 1 \leq \theta<\infty, \\
\|f\|_{B_{p, \infty}^{\Omega}}=\sup _{t>0} \frac{\Omega_{l}(f, t)_{p}}{\Omega(t)} .
\end{gathered}
$$

We note that, in the case $r=\left(r_{1}, \ldots, r_{d}\right), 0<r_{j}<l, j=\overline{1, d}$, and $\Omega(t)=\prod_{j=1}^{d} t_{j}^{r_{j}}$, the classes $B_{p, \theta}^{\Omega}$ are identical to analogs of the Besov classes $B_{p, \theta}^{r}$ which were considered in the papers [2,16]. In turn, for $\theta=\infty$ the classes $B_{p, \infty}^{r}=H_{p}^{r}$ are analogs of the Nikol'skii classes [18]. The classes $B_{p, \infty}^{\Omega}=H_{p}^{\Omega}$ were have studied in the paper by N.N. Pustovoitov [19].

In the following considerations we will use the definition of classes $B_{p, \theta}^{\Omega}$ in a slightly different form. To do this, we recall the definition of order relation. 
For two non-negative sequences $\left(a_{n}\right)_{n=1}^{\infty}$ and $\left(b_{n}\right)_{n=1}^{\infty}$ the relation (order inequality) $a_{n} \ll b_{n}$ means that there exists a constant $C_{3}>0$, which does not depend on $n$ and such that $a_{n} \leq C_{3} b_{n}$. The relation $a_{n} \asymp b_{n}$ is equivalent to $a_{n} \ll b_{n}$ and $b_{n} \ll a_{n}$.

To every vector $s \in \mathbb{N}^{d}$ we put the set

$$
\rho(s)=\left\{k \in \mathbb{Z}^{d}: 2^{s_{j}-1} \leq\left|k_{j}\right|<2^{s_{j}}, j=\overline{1, d}\right\}
$$

in correspondence, and, for $f \in L_{p}^{0}, 1<p<\infty$, we denote

$$
\delta_{s}(f):=\delta_{s}(f, x)=\sum_{k \in \rho(s)} \widehat{f}(k) e^{i(k, x)},
$$

where

$$
\widehat{f}(k)=(2 \pi)^{-d} \int_{\pi_{d}} f(t) e^{-i(k, t)} d t
$$

are the Fourier coefficients of the function $f$.

Therefore, for $f \in B_{p, \theta}^{\Omega}, 1<p<\infty, 1 \leq \theta \leq \infty$, where $\Omega(t)$ is a given function of the type of mixed modulus of continuity of order $l$, which satisfies conditions 1$)-4),\left(S^{\alpha}\right),\left(S_{l}\right)$, and the relations

$$
\|f\|_{B_{p, \theta}^{\Omega}} \asymp\left\{\begin{array}{cc}
\left(\sum_{s} \Omega^{-\theta}\left(2^{-s}\right)\left\|\delta_{s}(f)\right\|_{p}^{\theta}\right)^{1 / \theta}, & 1 \leq \theta<\infty, \\
\sup _{s} \frac{\left\|\delta_{s}(f)\right\|_{p}}{\Omega\left(2^{-s}\right)}, & \theta=\infty,
\end{array}\right.
$$

hold. Here and below, $\Omega\left(2^{-s}\right)=\Omega\left(2^{-s_{1}}, \ldots, 2^{-s_{d}}\right), s_{j} \in \mathbb{N}, j=\overline{1, d}$.

Note that the case $1 \leq \theta<\infty$ in (1) was considered in [37], and the case $\theta=\infty$ in [19].

For the norms of functions from the classes $B_{p, \theta}^{\Omega}$ for $p=1$ and $p=\infty$ we can write relations analogous to (1) by replacing the "blocks" $\delta_{s}(f)$ by others. Namely, by $V_{m}(t), m \in \mathbb{N}, t \in \mathbb{R}$, we denote the Vall'ee-Poussin kernel

$$
V_{m}(t)=1+2 \sum_{k=1}^{m} \cos k t+2 \sum_{k=m+1}^{2 m-1}\left(\frac{2 m-k}{m}\right) \cos k t
$$

(for the correctness of the definition of $V_{m}(t)$ we should assume that the last sum in this formula vanishes for $m=1$ ).

To every vector $s \in \mathbb{N}^{d}$, we put the polynomial

$$
A_{s}(x)=\prod_{j=1}^{d}\left(V_{2^{s_{j}}}\left(x_{j}\right)-V_{2^{s_{j}-1}}\left(x_{j}\right)\right), \quad x \in \mathbb{R}^{d},
$$

in correspondence, and, for $f \in L_{p}^{0}, 1 \leq p \leq \infty$, we set

$$
A_{s}(f):=A_{s}(f, x)=\left(f * A_{s}\right)(x),
$$

where $*$ means the convolution operation. Then the following relations hold:

$$
\|f\|_{B_{p, \theta}^{\Omega}} \asymp\left\{\begin{array}{cc}
\left(\sum_{s} \Omega^{-\theta}\left(2^{-s}\right)\left\|A_{s}(f)\right\|_{p}^{\theta}\right)^{1 / \theta}, & 1 \leq \theta<\infty, \\
\sup _{s} \frac{\left\|A_{s}(f)\right\|_{p}}{\Omega\left(2^{-s}\right)}, & \theta=\infty .
\end{array}\right.
$$


Note that the case $1 \leq \theta<\infty$ in (2) was considered in [30], and the case $\theta=\infty$ in [19].

In the following research, we consider the classes $B_{p, \theta}^{\Omega}$ defined by a function of the type of a mixed modulus of continuity of order $l$ of the special form

$$
\Omega(t)=\omega\left(\prod_{j=1}^{d} t_{j}\right),
$$

where $\omega(\tau)$ is a given function (of one variable) of the type of a modulus of continuity of order $l$ that satisfies conditions $\left(S^{\alpha}\right)$ and $\left(S_{l}\right)$.

It is easy to verify that the function $\Omega(t)$ of the form (3) satisfies properties 1) - 4) of a function of the type of mixed modulus of continuity of order $l$ and satisfies conditions $\left(S^{\alpha}\right)$ and $\left(S_{l}\right)$. Therefore, the above mentioned relations (1), (2) for the norms of functions of the class $B_{p, \theta}^{\Omega}$ remain true.

Now we define the norm in the subspace $B_{\infty, 1}$. For any trigonometric polynomial $t$, it is defined by the formula

$$
\|t\|_{B_{\infty, 1}}=\sum_{s \in \mathbb{N}^{d} \cup\{0\}}\left\|A_{s}(t)\right\|_{\infty} .
$$

In the same way, the norm $\|f\|_{B_{\infty, 1}}$ for the functions $f \in L_{1}$ under the condition of convergence of the series $\sum_{s \in \mathbb{N}^{d} \cup\{0\}}\left\|A_{s}(f)\right\|_{\infty}$ is defined. We note that, in this case, the relation

$$
\|\cdot\|_{\infty} \leq\|\cdot\|_{B_{\infty, 1}}
$$

holds.

\section{APPROXIMATIVE CHARACTERISTICS AND AUXILIARY STATEMENTS}

Let $\left\{u_{i}\right\}_{i=1}^{M}$ be an orthonormal in the space $L_{2}\left(\pi_{d}\right)$ system of functions $u_{i} \in L_{\infty}\left(\pi_{d}\right), i=$ $\overline{1, M}$. For every function $f \in L_{q}\left(\pi_{d}\right), 1 \leq q \leq \infty$, we put an approximative aggregate of the form $\sum_{i=1}^{M}\left(f, u_{i}\right) u_{i}$ in correspondence, $i$. e., the orthogonal projection of the function $f$ onto the subspace generated by the system of functions $\left\{u_{i}\right\}_{i=1}^{M}$. Here and below, we set

$$
\left(f, u_{i}\right)=(2 \pi)^{-d} \int_{\pi_{d}} f(x) \bar{u}_{i}(x) d x .
$$

Then, for the functional class $F \subset L_{q}\left(\pi_{d}\right)$, the quantity

$$
d_{M}^{\perp}\left(F, L_{q}\right)=\inf _{\left\{u_{i}\right\}_{i=1}^{M}} \sup _{f \in F}\left\|f-\sum_{i=1}^{M}\left(f, u_{i}\right) u_{i}\right\|_{q}
$$

is called the orthowidth (the Fourier-width) of this class in the space $L_{q}\left(\pi_{d}\right)$. The orthowidth $d_{M}^{\perp}\left(F, L_{q}\right)$ introduced by V.N. Temlyakov [35].

In addition, in [35] V.N. Temlyakov considered similar to the Fourier-width quantity of $d_{M}^{B}\left(F, L_{q}\right)$, which is defined as follows

$$
d_{M}^{B}\left(F, L_{q}\right)=\inf _{G \in L_{M}(B)_{q}} \sup _{f \in F \cap D(G)}\|f-G f\|_{q} .
$$


Here by $L_{M}(B)_{q}$ we denote the set of linear operators satisfying the conditions:

a) the domain of definition $D(G)$ of these operators contains all trigonometric polynomials, and their domain of values is contained in a subspace with dimension $M$ of the space $L_{q}\left(\pi_{d}\right)$;

b) there exists a number $B \geq 1$ such that, for all vectors $k=\left(k_{1}, \ldots, k_{d}\right), k_{j} \in \mathbb{Z}, j=\overline{1, d}$, the inequality $\left\|G e^{i(k, \cdot)}\right\|_{2} \leq B$ holds.

We note that $L_{M}(1)_{2}$ contains the operators of orthogonal projection onto the spaces of dimension $M$ as well as operators defined on an orthonormal system of functions involving a multiplier defined by a sequence $\left\{\lambda_{m}\right\}$ such that $\left|\lambda_{m}\right| \leq 1$ for all $m$.

From (5) and (6), it is easy to see that the quantities $d_{M}^{\perp}\left(F, L_{q}\right)$ and $d_{M}^{B}\left(F, L_{q}\right)$ are connected with each other by the inequality

$$
d_{M}^{B}\left(F, L_{q}\right) \leq d_{M}^{\perp}\left(F, L_{q}\right)
$$

It is obvious that such a relation also holds for case in which instead of the space $L_{q}$ the space $B_{\infty, 1}$ is considered, i. e.

$$
d_{M}^{B}\left(F, B_{\infty, 1}\right) \leq d_{M}^{\perp}\left(F, B_{\infty, 1}\right)
$$

The quantities (5) and (6) for various functional classes $F$, both in Lebesgue spaces $L_{q}\left(\pi_{d}\right)$, $1 \leq q \leq \infty$, and in other functional spaces were investigated in $[1,3,4,6,9,12,13,20,24-26,30$, $35,36]$. A more detailed bibliography can be found in the monographs $[10,23,32,33]$.

We recall some of the well-known statements that will be used in the subsequent considerations.

Theorem 1 ([11]). Let $1 \leq p<\infty, 1 \leq \theta \leq \infty, \Omega(t)=\omega\left(\prod_{j=1}^{d} t_{j}\right)$, where $\omega$ satisfies condition $\left(S^{\alpha}\right)$ with some $\alpha>1 / p$ and condition $\left(S_{l}\right)$. Then for any $M, n \in \mathbb{N}$, such that $M \asymp 2^{n} n^{d-1}$, the relations

$$
d_{M}^{\perp}\left(B_{p, \theta}^{\Omega}, L_{\infty}\right) \asymp d_{M}^{B}\left(B_{p, \theta}^{\Omega}, L_{\infty}\right) \asymp \omega\left(2^{-n}\right) 2^{n / p} n^{(d-1)(1-1 / \theta)}
$$

hold.

Theorem 2 ([11]). Let $1 \leq \theta \leq \infty, \Omega(t)=\omega\left(\prod_{j=1}^{d} t_{j}\right)$, where $\omega$ satisfies condition $\left(S^{\alpha}\right)$ with some $\alpha>0$ and condition $\left(S_{l}\right)$. Then for any $M, n \in \mathbb{N}$, such that $M \asymp 2^{n} n^{d-1}$, the relations

$$
d_{M}^{B}\left(B_{\infty, \theta}^{\Omega}, L_{\infty}\right) \asymp \omega\left(2^{-n}\right) n^{(d-1)(1-1 / \theta)}
$$

hold.

To formulate the following statement, we need the appropriate notation.

For $d \geq 2$ and $n \in \mathbb{N}$ we denote by $Q_{n}$ the set

$$
Q_{n}:=\bigcup_{(s, 1)<n} \rho(s),
$$

which is called a step hyperbolic cross.

Accordingly, for the function $f \in L_{1}\left(\pi_{d}\right)$ we denote by $S_{Q_{n}}(f)$ its step hyperbolic Fourier sum

$$
S_{Q_{n}}(f):=\sum_{k \in Q_{n}} \widehat{f}(k) e^{i(k, x)}
$$


and for the functional class $F \subset X$ we put

$$
\mathcal{E}_{Q_{n}}(F)_{X}:=\sup _{f \in F}\left\|f-S_{Q_{n}}(f)\right\|_{X} .
$$

In the one-dimensional case, instead of $\mathcal{E}_{Q_{n}}(F)_{X}$ we use the notation $\mathcal{E}_{n}(F)_{X}$, i. e.

$$
\mathcal{E}_{n}(F)_{X}:=\sup _{f \in F}\left\|f-S_{n}(f)\right\|_{X}
$$

where

$$
S_{n}(f):=\sum_{k=-2^{n}+1}^{2^{n}} \widehat{f}(k) e^{i k x}
$$

The following statement is obtained in [29].

Theorem 3. Let $d \geq 1,1<p<\infty, 1 \leq \theta \leq \infty, \Omega(t)=\omega\left(\prod_{j=1}^{d} t_{j}\right)$, where $\omega$ satisfies condition $\left(S^{\alpha}\right)$ with some $\alpha>1 / p$ and condition $\left(S_{l}\right)$. Then

$$
\mathcal{E}_{Q_{n}}\left(B_{p, \theta}^{\Omega}\right)_{\infty} \asymp \omega\left(2^{-n}\right) 2^{n / p} n^{(d-1)(1-1 / \theta)} .
$$

\section{ESTIMATES OF APPROXIMATIVE CHARACTERISTICS}

The following theorem is true.

Theorem 4. Let $d \geq 1,1<p<\infty, 1 \leq \theta \leq \infty, \Omega(t)=\omega\left(\prod_{j=1}^{d} t_{j}\right)$, where $\omega$ satisfies condition $\left(S^{\alpha}\right)$ with some $\alpha>1 / p$ and condition $\left(S_{l}\right)$. Then for any $M, n \in \mathbb{N}$, such that $M \asymp 2^{n} n^{d-1}$, the relations

$$
d_{M}^{\perp}\left(B_{p, \theta}^{\Omega}, B_{\infty, 1}\right) \asymp d_{M}^{B}\left(B_{p, \theta}^{\Omega}, B_{\infty, 1}\right) \asymp \omega\left(2^{-n}\right) 2^{n / p_{n}}(d-1)(1-1 / \theta)
$$

hold.

Proof. According to the inequality (7), to prove the relations (9), it is sufficient to establish the lower bound for the quantity $d_{M}^{B}\left(B_{p, \theta}^{\Omega}, B_{\infty, 1}\right)$ and the upper bound for the orthowidth $d_{M}^{\perp}\left(B_{p, \theta}^{\Omega}, B_{\infty, 1}\right)$.

The lower bound for the quantity $d_{M}^{B}\left(B_{p, \theta}^{\Omega}, B_{\infty, 1}\right)$ is a consequence of Theorem 1 due to the relation (4). The corresponding upper bound for the orthowidth is obtained as a consequence of Theorem 3.

Thus, choosing the number $n \in \mathbb{N}$ according to the condition $M \asymp 2^{n} n^{d-1}$, we can write

$$
d_{M}^{\perp}\left(B_{p, \theta}^{\Omega}, B_{\infty, 1}\right) \ll \mathcal{E}_{Q_{n}}\left(B_{p, \theta}^{\Omega}\right)_{B_{\infty, 1}} \asymp \omega\left(2^{-n}\right) 2^{n / p} n^{(d-1)(1-1 / \theta)} .
$$

The formulation of Theorem 4 does not contain the cases $p=1$ and $p=\infty$, where we managed to establish only the order of the quantity $d_{M}^{B}\left(B_{p, \theta}^{\Omega}, B_{\infty, 1}\right), p \in\{1, \infty\}$.

Theorem 5. Let $d \geq 2,1 \leq \theta \leq \infty, \Omega(t)=\omega\left(\prod_{j=1}^{d} t_{j}\right)$, where $\omega$ satisfies condition $\left(S^{\alpha}\right)$ with some $\alpha>1$ and condition $\left(S_{l}\right)$. Then for any $M, n \in \mathbb{N}$, such that $M \asymp 2^{n} n^{d-1}$, the relation

$$
d_{M}^{B}\left(B_{1, \theta}^{\Omega}, B_{\infty, 1}\right) \asymp \omega\left(2^{-n}\right) 2^{n} n^{(d-1)(1-1 / \theta)}
$$

holds. 
Proof. Firstly, we establish the upper bound in (10). For this purpose, we choose the number $n \in \mathbb{N}$ according to the condition $M \asymp 2^{n} n^{d-1}$ and consider an approximation of the functions $f \in B_{1, \theta}^{\Omega}$ by polynomials of the form

$$
t_{n}(f)=\sum_{(s, 1)<n} A_{s}(f)
$$

As it was noted above, the operator $G$, which puts into the correspondence to the function $f$ a polynomial of this form, belongs to $L_{M}(1)_{2}$. Then, according to the definition of the norm in the space $B_{\infty, 1}$ and the convolution property, we can write

$$
\begin{aligned}
\left\|f-t_{n}(f)\right\|_{B_{\infty, 1}}=\left\|\sum_{(s, 1) \geq n} A_{s}(f)\right\|_{B_{\infty}, 1}=\sum_{s}\left\|A_{s} * \sum_{\substack{s^{\prime} \in \mathbb{N}^{d} \\
\left(s^{\prime}, 1\right) \geq n}} A_{s^{\prime}}(f)\right\|_{\infty} \\
\leq \sum_{(s, 1) \geq n-d}\left\|A_{s} * \sum_{\left\|s-s^{\prime}\right\|_{\infty} \leq 1} A_{s^{\prime}}(f)\right\|_{\infty} \leq \sum_{(s, 1) \geq n-d}\left\|A_{s}\right\|_{\infty}\left\|_{\left\|s-s^{\prime}\right\|_{\infty} \leq 1} A_{s^{\prime}}(f)\right\|_{1}=I_{1} .
\end{aligned}
$$

Using the relation (see example [32, Ch.2, §5])

$$
\left\|A_{s}\right\|_{p} \asymp 2^{(s, 1)(1-1 / p)}, \quad 1 \leq p \leq \infty,
$$

we continue the estimate as follows

$$
\begin{aligned}
I_{1} \asymp \sum_{(s, 1) \geq n-d} 2^{(s, 1)} \sum_{\left\|s-s^{\prime}\right\|_{\infty} \leq 1}\left\|A_{s^{\prime}}(f)\right\|_{1} \ll \sum_{(s, 1) \geq n-2 d} 2^{(s, 1)}\left\|A_{s}(f)\right\|_{1} \\
=\sum_{(s, 1) \geq n-2 d} \omega^{-1}\left(2^{-(s, 1)}\right)\left\|A_{s}(f)\right\|_{1} \omega\left(2^{-(s, 1)}\right) 2^{(s, 1)}=I_{2} .
\end{aligned}
$$

Let us consider several cases depending on the values of parameter $\theta$.

1. Let $\theta \in(1, \infty)$. Applying the Hölder's inequality, we obtain

$$
\begin{aligned}
I_{2} & \leq\left(\sum_{(s, 1) \geq n-2 d} \omega^{-\theta}\left(2^{-(s, 1)}\right)\left\|A_{s}(f)\right\|_{1}^{\theta}\right)^{1 / \theta}\left(\sum_{(s, 1) \geq n-2 d} \omega^{\theta^{\prime}}\left(2^{-(s, 1)}\right) 2^{(s, 1) \theta^{\prime}}\right)^{1 / \theta^{\prime}} \\
& \ll\|f\|_{B_{1, \theta}^{\Omega}}\left(\sum_{(s, 1) \geq n-2 d} \omega^{\theta^{\prime}}\left(2^{-(s, 1)}\right) 2^{(s, 1) \theta^{\prime}}\right)^{1 / \theta^{\prime}} \leq\left(\sum_{(s, 1) \geq n-2 d} \omega^{\theta^{\prime}}\left(2^{-(s, 1)}\right) 2^{-(s, 1) \theta^{\prime}}\right)^{1 / \theta^{\prime}}=I_{3},
\end{aligned}
$$

where $1 / \theta+1 / \theta^{\prime}=1$.

We denote $n-2 d=m$. Taking into account that for $(s, 1) \geq m$

$$
\frac{\omega\left(2^{-(s, 1)}\right)}{2^{-\alpha(s, 1)}} \leq C_{4} \frac{\omega\left(2^{-m}\right)}{2^{-\alpha m}}, \quad C_{4}>0,
$$

we get

$$
\begin{aligned}
I_{3} & \ll \frac{\omega\left(2^{-m}\right)}{2^{-\alpha m}}\left(\sum_{(s, 1) \geq m} 2^{-(s, 1)(\alpha-1) \theta^{\prime}}\right)^{1 / \theta^{\prime}}=\frac{\omega\left(2^{-m}\right)}{2^{-\alpha m}}\left(\sum_{j \geq m} 2^{-j(\alpha-1) \theta^{\prime}} \sum_{(s, 1)=j} 1\right)^{1 / \theta^{\prime}} \\
& \ll \frac{\omega\left(2^{-m}\right)}{2^{-\alpha m}} 2^{-m(\alpha-1)} m^{(d-1) / \theta^{\prime}}=\omega\left(2^{-m}\right) 2^{m} m^{(d-1)(1-1 / \theta)} .
\end{aligned}
$$


2. Let $\theta=1$. In this case we have

$$
\begin{aligned}
I_{2} & \leq \sup _{s:(s, 1) \geq m} \omega\left(2^{-(s, 1)}\right) 2^{(s, 1)} \sum_{(s, 1) \geq m} \omega^{-1}\left(2^{-(s, 1)}\right)\left\|A_{s}(f)\right\|_{1} \\
& \ll \omega\left(2^{-m}\right) 2^{m}\|f\|_{B_{1,1}^{\Omega}} \ll \omega\left(2^{-m}\right) 2^{m} .
\end{aligned}
$$

3. If $\theta=\infty$, then for the quantity $I_{2}$ we can write

$$
\begin{aligned}
I_{2} \leq \sup _{s:(s, 1) \geq m} \frac{\left\|A_{s}(f)\right\|_{1}}{\omega\left(2^{-(s, 1)}\right)} \sum_{(s, 1) \geq m} \omega\left(2^{-(s, 1)}\right) 2^{(s, 1)} & \ll\|f\|_{B_{1, \infty}^{\Omega}} \sum_{(s, 1) \geq m} \frac{\omega\left(2^{-(s, 1)}\right)}{2^{-\alpha(s, 1)}} 2^{-(\alpha-1)(s, 1)} \\
& \ll \frac{\omega\left(2^{-m}\right)}{2^{-\alpha m}} 2^{-(\alpha-1) m} m^{d-1}=\omega\left(2^{-m}\right) 2^{m} m^{d-1} .
\end{aligned}
$$

Thus, taking into account the relations (12)-(17), we obtain the required upper bound for the quantity $d_{M}^{B}\left(B_{1, \theta}^{\Omega}, B_{\infty, 1}\right)$.

The corresponding lower bound in (10) follows from Theorem 1 according to the relation (4).

With a certain modification of the considerations, it is easy to establish an analogue of Theorem 5 for the one-dimensional case. The corresponding statement is as follows.

Theorem 6. Let $d=1,1 \leq \theta \leq \infty$, and $\omega$ satisfies condition $\left(S^{\alpha}\right)$ with some $\alpha>1$ and condition $\left(S_{l}\right)$. Then the relation

$$
d_{M}^{B}\left(B_{1, \theta}^{\omega}, B_{\infty, 1}\right) \asymp \omega\left(M^{-1}\right) M
$$

holds.

Now we establish the order estimate of the quantity $d_{M}^{B}\left(B_{\infty, \theta}^{\Omega}, B_{\infty, 1}\right)$.

Theorem 7. Let $d \geq 2,1 \leq \theta \leq \infty, \Omega(t)=\omega\left(\prod_{j=1}^{d} t_{j}\right)$, where $\omega$ satisfies condition $\left(S^{\alpha}\right)$ with some $\alpha>0$ and condition $\left(S_{l}\right)$. Then for any $M, n \in \mathbb{N}$, such that $M \asymp 2^{n} n^{d-1}$, the relation

$$
d_{M}^{B}\left(B_{\infty, \theta}^{\Omega}, B_{\infty, 1}\right) \asymp \omega\left(2^{-n}\right) n^{(d-1)(1-1 / \theta)}
$$

holds.

Proof. Similarly as in the proof of Theorem 5, the upper bound is obtained by approximating the functions $f \in B_{\infty, \theta}^{\Omega}$ by trigonometric polynomials of the form (11) under the condition $M \asymp 2^{n} n^{d-1}$. The corresponding estimate is obtained in [15].

Regarding the lower bound in (18), we note that it is a consequence of the Theorem 2 according to the inequality (4).

The corresponding statement for the one-dimensional case is as follows.

Theorem 8. Let $d=1,1 \leq \theta \leq \infty$, and $\omega$ satisfies condition $\left(S^{\alpha}\right)$ with some $\alpha>0$ and condition $\left(S_{l}\right)$. Then the relation

$$
d_{M}^{B}\left(B_{\infty, \theta}^{\omega}, B_{\infty, 1}\right) \asymp \omega\left(M^{-1}\right)
$$

holds.

Remark 1. Analyzing the results obtained in this part of the paper, we get the following conclusion: in the one-dimensional case, in contrast to the multidimensional one, the obtained estimates of the corresponding approximative characteristics are independent of the parame$\operatorname{ter} \theta$. 


\section{ESTIMATES OF NORMS OF APPROXIMATION OPERATORS}

Firstly, we make some remarks and introduce necessary notation. Let $Q_{n}$ be a set denoted by (8) and

$$
T\left(Q_{n}\right)=\left\{t: t(x)=\sum_{k \in Q_{n}} c_{k} e^{i(k, x)}, x \in \mathbb{R}^{d}\right\} .
$$

Let $X \in L_{1}\left(\pi_{d}\right)$ be some normed functional space with norm $\|\cdot\|_{X}$.

For $f \in X$ we denote the quantity

$$
E_{Q_{n}}(f)_{X}:=\inf _{t \in T\left(Q_{n}\right)}\|f-t\|_{X}
$$

and for the class $F \subset X$ we put

$$
E_{Q_{n}}(F)_{X}:=\sup _{f \in F} E_{Q_{n}}(f)_{X}
$$

The quantities $E_{Q_{n}}(f)_{X}$ and $E_{Q_{n}}(F)_{X}$ are called the best approximations in the space $X$ respectively of the function $f \in X$ and of the class $F$ by trigonometric polynomials from the set $T\left(Q_{n}\right)$.

In the one-dimensional case, the corresponding approximative characteristics are defined as follows.

Let $X \in L_{1}\left(\pi_{1}\right)$ be a normed functional space with norm $\|\cdot\|_{X}$ and $T\left(2^{n}\right), n \in \mathbb{N}$, be the set of trigonometric polynomials of the form

$$
T\left(2^{n}\right)=\left\{t: t(x)=\sum_{k=-2^{n}+1}^{2^{n}-1} c_{k} e^{i k x}, x \in \mathbb{R}\right\} .
$$

Then for the function $f \in X$ by $E_{2^{n}}(f)_{X}$ we denote the quantity of its best approximation by polynomials from the set $T\left(2^{n}\right)$, i.e.

$$
E_{2^{n}}(f)_{X}=\inf _{t \in T\left(2^{n}\right)}\|f-t\|_{X}
$$

and for the functional class $F \subset X$ we put

$$
E_{2^{n}}(F)_{X}:=\sup _{f \in F} E_{2^{n}}(f)_{X} .
$$

The following statement is established in the paper [15].

Theorem 9. Let $d \geq 2,1 \leq \theta \leq \infty, \Omega(t)=\omega\left(\prod_{j=1}^{d} t_{j}\right)$, where $\omega$ satisfies condition $\left(S^{\alpha}\right)$ with some $\alpha>0$ and condition $\left(S_{l}\right)$. Then

$$
E_{Q_{n}}\left(B_{\infty, \theta}^{\Omega}\right)_{B_{\infty, 1}} \asymp \omega\left(2^{-n}\right) n^{(d-1)(1-1 / \theta)} .
$$

It is important to note that the estimate (19) is obtained by approximation using the linear method. More specifically, such a method in [15] used a sequence of linear operators $\left\{\mathbb{V}_{Q_{n}}\right\}_{n=1}^{\infty}$, which to the function $f \in B_{\infty, \theta}^{\Omega}$ put in correspondence the polynomial of the form

$$
\mathbb{V}_{Q_{n}} f=V_{Q_{n}}(f)=\sum_{(s, 1)<n} A_{s}(f)=f * V_{Q_{n}}
$$


where

$$
V_{Q_{n}}:=V_{Q_{n}}(x)=\sum_{(s, 1)<n} A_{s}(x) .
$$

However, the sequence of operators $\left\{\mathbb{V}_{Q_{n}}\right\}_{n=1}^{\infty}$ has one significant drawback, that is that the norm of the operator $\mathbb{V}_{Q_{n}}$, as an operator going from $L_{\infty}$ to $L_{\infty}$ is equal to $\left\|V_{Q_{n}}\right\|_{1}$ and thus, (see consequence [32, Theorem 1.2.1])

$$
\left\|V_{Q_{n}}\right\|_{1} \gg n^{d-1}
$$

In other words, the sequence of norms of linear operators $\left\{\mathbb{V}_{Q_{n}}\right\}_{n=1}^{\infty}$, which realize the order of quantity $E_{Q_{n}}\left(B_{\infty, \theta}^{\Omega}\right)_{B_{\infty, 1}}$ for $d \geq 2$, is unbounded.

Therefore, the question naturally arises about the existence of a sequence of linear operators $\mathcal{L}_{Q_{n}}: L_{\infty} \rightarrow T\left(Q_{n}\right)$ with bounded norms $\left\|\mathcal{L}_{Q_{n}}\right\|$, which well approximate the classes $B_{\infty, \theta}^{\Omega}$ in the space $B_{\infty, 1}$.

The answer to this question is contained in the following two statements in multidimensional $(d \geq 2)$ and one-dimensional cases, respectively.

Theorem 10. Assume that on $L_{\infty}^{0}\left(\pi_{d}\right), d \geq 2$, a sequence of bounded linear operators $\mathcal{L}_{Q_{n}}$ is defined, that to every function from $L_{\infty}^{0}\left(\pi_{d}\right)$ put in correspondence the trigonometric polynomial from the set $T_{Q_{n}}$, so that for the functions $f \in B_{\infty, \theta}^{\Omega}, 1 \leq \theta \leq \infty, \Omega(t)=\omega\left(\prod_{j=1}^{d} t_{j}\right)$, where $\omega$ satisfies the conditions $\left(S^{\alpha}\right), \alpha>0$ and $\left(S_{l}\right)$, the order inequality

$$
\left\|f-\mathcal{L}_{Q_{n}}(f)\right\|_{B_{\infty, 1}} \ll E_{Q_{n}}\left(B_{\infty, \theta}^{\Omega}\right)_{B_{\infty, 1}}
$$

holds.

Then for any $\varepsilon>0$ the following estimate is true

$$
\left\|\mathcal{L}_{Q_{n}}\right\| \gg n^{(d-1)(1-\varepsilon)} .
$$

Proof. Let $\tau=\left(\tau_{1}, \ldots, \tau_{d}\right), \tau_{j} \in \mathbb{R}, j=\overline{1, d}$, and $I_{\tau}$ denotes a shift operator of the argument of the function $f$ to the vector $\tau$, i. e. $I_{\tau} f(x)=f(x+\tau)$. Following J. Marcinkiewicz [17], consider the bounded linear operator $T_{Q_{n}}$ defined by the equality

$$
\left(T_{Q_{n}} f\right)(x)=(2 \pi)^{-d} \int_{\pi_{d}}\left(I_{-\tau} \mathcal{L}_{Q_{n}} I_{\tau} f\right)(x) d \tau .
$$

Then, due to the invariance of the norm for the shift of the argument, for the norms of the operators $T_{Q_{n}}$ and $\mathcal{L}_{Q_{n}}$ the relation

$$
\left\|T_{Q_{n}}\right\| \leq\left\|\mathcal{L}_{Q_{n}}\right\|
$$

is valid.

It is also easy to see (see example [21]) that the operator $T_{Q_{n}}$ acts on the function $f$ as a convolution operator, i.e.

$$
\left(T_{Q_{n}} f\right)(x)=f(x) * \sum_{k \in Q_{n}} c_{n, k} e^{i(k, x)} .
$$


Next, let $f \in B_{\infty, \theta}^{\Omega}$. Then $I_{\tau} f \in B_{\infty, \theta}^{\Omega}$ and according to the condition of the theorem we get

$$
\left\|I_{\tau} f-\mathcal{L}_{Q_{n}}\left(I_{\tau} f\right)\right\|_{B_{\infty, 1}} \ll E_{Q_{n}}\left(B_{\infty, \theta}^{\Omega}\right)_{B_{\infty, 1}} .
$$

Let $I$ be the identical operator. Then, using (22), we have

$$
\begin{aligned}
\left\|f-T_{Q_{n}}(f)\right\|_{B_{\infty, 1}} & =(2 \pi)^{-d}\left\|\int_{\pi_{d}} I_{-\tau}\left(I-\mathcal{L}_{Q_{n}}\right)\left(I_{\tau} f\right) d \tau\right\|_{B_{\infty, 1}} \\
& \leq(2 \pi)^{-d} \int_{\pi_{d}}\left\|I_{-\tau}\left(I_{\tau}(f)-\mathcal{L}_{Q_{n}}\left(I_{\tau}(f)\right)\right)\right\|_{B_{\infty, 1}} d \tau \\
& =(2 \pi)^{-d} \int_{\pi_{d}}\left\|I_{\tau}(f)-\mathcal{L}_{Q_{n}}\left(I_{\tau}(f)\right)\right\|_{B_{\infty, 1}} d \tau \ll E_{Q_{n}}\left(B_{\infty, \theta}^{\Omega}\right)_{B_{\infty, 1}} .
\end{aligned}
$$

Thus, it follows from relations (20) and (23), that it is sufficient to prove the theorem for the operator acting according to the formula (21).

Let

$$
\mathcal{L}_{Q_{n}}(f)=(2 \pi)^{-d} \int_{\pi_{d}} f(x-y) L_{Q_{n}}(y) d y,
$$

where

$$
L_{Q_{n}}(y)=\sum_{k \in Q_{n}} c_{n, k} e^{i(k, y)}
$$

In this case, for the norm of the operator $\mathcal{L}_{Q_{n}}$ we have $\left\|\mathcal{L}_{Q_{n}}\right\|=\left\|L_{Q_{n}}\right\|_{1}$.

For further consideration, we need an auxiliary statement.

Lemma 1. There exists $\delta>0$ such that inequality

$$
\sum_{k \in Q_{n}}\left|c_{n, k}\right| \geq \delta\left|Q_{n}\right|
$$

holds for all $n$.

Proof. We use the method of the opposite, i.e. assume that for any $\delta>0$ there exists $n$ such that

$$
\sum_{k \in Q_{n}}\left|c_{n, k}\right|<\delta\left|Q_{n}\right|
$$

Denote by $S$ the set of such vectors $s=\left(s_{1}, \ldots, s_{d}\right),(s, 1) \leq n, \rho(s) \in Q_{n}$, that for some vector $k^{s}=\left(k_{1}^{s}, \ldots, k_{d}^{s}\right) \in \rho(s)$ the inequality $\left|c_{n, k^{s}}\right| \leq 1 / 2$ holds. In [21] it is established that for the number of elements of the set $S$ the following estimate is true

$$
|S| \gg n^{d-1} \log _{2} \frac{1}{\delta}
$$

Let us consider the function

$$
f_{1}(x)=C_{5}|S|^{-1 / \theta} \sum_{s \in S} \omega\left(2^{-\|s\|_{1}}\right) e^{i\left(k^{s}, x\right)}, \quad 1 \leq \theta \leq \infty, C_{5}>0,
$$

where the vectors $k^{s}=\left(k_{1}^{s}, \ldots, k_{d}^{s}\right) \in Q_{n}$ are defined above. 
It is easy to prove that this function with the corresponding constant $C_{5}>0$ belongs to the class $B_{\infty, \theta}^{\Omega}, 1 \leq \theta \leq \infty$.

Next we need the estimation obtained in [15], namely

$$
E_{Q_{n}}\left(B_{\infty, \theta}^{\Omega}\right)_{B_{\infty, 1}} \asymp \omega\left(2^{-n}\right) n^{(d-1)(1-1 / \theta)}, \quad 1 \leq \theta \leq \infty .
$$

Since for the function $f_{1}$ the relation

$$
\left\|f_{1}-\mathcal{L}_{Q_{n}}\left(f_{1}\right)\right\|_{B_{\infty, 1}} \ll E_{Q_{n}}\left(B_{\infty, \theta}^{\Omega}\right)_{B_{\infty, 1}}
$$

is fulfilled, according to (25) and (26) we get

$$
\left\|f_{1}-\mathcal{L}_{Q_{n}}\left(f_{1}\right)\right\|_{B_{\infty, 1}} \ll \omega\left(2^{-n}\right) n^{(d-1)(1-1 / \theta)}, \quad 1 \leq \theta \leq \infty .
$$

Now we establish the lower bound of left part in (20). We have

$$
\begin{aligned}
\left\|f_{1}-\mathcal{L}_{Q_{n}}\left(f_{1}\right)\right\|_{B_{\infty, 1}} & \geq\left\|f_{1}-\mathcal{L}_{Q_{n}}\left(f_{1}\right)\right\|_{\infty} \geq\left|f_{1}(0)-\mathcal{L}_{Q_{n}}\left(f_{1}(0)\right)\right| \\
& =C_{5}|S|^{-1 / \theta}\left|\sum_{s \in S}\left(1-c_{n, k^{s}}\right) \omega\left(2^{-\|s\|_{1}}\right)\right| \\
& \gg|S|^{-1 / \theta} \sum_{s \in S} \omega\left(2^{-\|s\|_{1}}\right) \gg \omega\left(2^{-n}\right)|S|^{-1 / \theta}|S|=\omega\left(2^{-n}\right)|S|^{1-1 / \theta} .
\end{aligned}
$$

From (27) and (28) we get the relation

$$
\omega\left(2^{-n}\right)|S|^{1-1 / \theta} \ll \omega\left(2^{-n}\right) n^{(d-1)(1-1 / \theta)} ;
$$

it follows the estimation $|S| \ll n^{d-1}$.

By comparing (24), we get

$$
n^{d-1} \log _{2} \frac{1}{\delta} \ll n^{d-1}
$$

so

$$
\log _{2} \frac{1}{\delta} \leq C_{6}
$$

where $C_{6}$ is some constant.

It is easy to see that for some $\delta>0$ this inequality is false.

To complete the proof of Theorem 10, we need the following auxiliary statement [34].

Lemma 2. For each $\eta>0$ there exists a constant $C(\eta)$ such that for any polynomial $t \in T\left(Q_{n}\right)$ the following inequality holds

$$
\sum_{k \in Q_{n}}|\widehat{t}(k)| \leq C(\eta) n^{\eta} 2^{n}\|t\|_{1}
$$

So by submitting (29) as

$$
\|t\|_{1} \geq C(\delta) n^{-\delta} 2^{-n} \sum_{k \in Q_{n}}|\widehat{t}(k)|
$$

and using Lemma 1, where the number $\delta>0$ satisfies its conditions, we have

$$
\left\|\mathcal{L}_{Q_{n}}\right\|=\left\|L_{Q_{n}}\right\|_{1} \gg n^{-\delta} 2^{-n}\left|Q_{n}\right| \asymp n^{-\delta} 2^{-n} 2^{n} n^{d-1}=n^{(d-1)(1-\delta /(d-1))} .
$$

Now we put $\varepsilon=\delta /(d-1)$ and with (30) we get the necessary estimate. 
Thus, as the obtained result shows, in the case of $d \geq 2$ the norms of sequences of operators $\left\{\mathcal{L}_{Q_{n}}\right\}_{n=1}^{\infty}$, which realize the orders of the best approximations of the classes $B_{\infty, \theta}^{\Omega}$ in the space $B_{\infty, 1}$ by trigonometric polynomials from the set $T\left(Q_{n}\right)$, are unbounded. In this regard, it should be noted that in the one-dimensional case, the situation is completely different.

We recall that in the paper [15] the following statement was established.

Theorem 11. Let $d=1,1 \leq \theta \leq \infty$, and $\omega$ satisfies condition $\left(S^{\alpha}\right)$ with some $\alpha>0$ and condition $\left(S_{l}\right)$. Then

$$
E_{2^{n}}\left(B_{\infty, \theta}^{\omega}\right)_{B_{\infty, 1}} \asymp \omega\left(2^{-n}\right) .
$$

It should be noted that the estimate (31) is realized by approximation by trigonometric polynomials

$$
t_{n}(f):=t_{n}(f, x)=\sum_{s=1}^{n-1} A_{s}(f, x), \quad x \in \mathbb{R}, f \in B_{\infty, \theta}^{\omega},
$$

which are generated by the operators $\mathbb{A}_{n}$, acting by the formula

$$
\mathbb{A}_{n} f=\tau_{n}(f) .
$$

But it is easy to see that the sequence of norms of the operators $\left\{\mathbb{A}_{n}\right\}_{n=1}^{\infty}$ is bounded because

$$
\left\|\mathbb{A}_{n}\right\|=\left\|\sum_{s=1}^{n-1} A_{s}\right\|_{1} \leq\left\|V_{1}\right\|_{1}+\left\|V_{n-1}\right\|_{1} \leq C_{7}, \quad C_{7}>0 .
$$

Remark 2. In the case of $\Omega(t)=\prod_{j=1}^{d} t_{j}^{r_{j}}$, i.e. for the classes $B_{p, \theta}^{r}$ similar problems were studied in [22].

\section{REFERENCES}

[1] Akishev G.A. The ortho-diameters of Nikol'skii and Besov classes in the Lorentz spaces. Russian Math. (Iz. VUZ) 2009, 53 (2), 21-29. doi:10.3103/S1066369X09020029 (translation of Izv. Vyssh. Uchebn. Zaved. Mat. 2009, 2, 25-33. (in Russian))

[2] Amanov T.I. Representation and embedding theorems for function spaces $S_{p, \theta}^{(r)} B\left(\mathbb{R}_{n}\right)$ and $S_{p, \theta^{*}}^{(r)} B,\left(0 \leq x_{j} \leq 2 \pi ; j=\right.$ $1, \ldots, n)$. Tr. Mat. Inst. Steklova 1965, 77, 5-34. (in Russian)

[3] Andrianov A.V., Temlyakov V.N. On Two Methods of Generalization of Properties of Univariate Function Systems to Their Tensor Product. Proc. Steklov Inst. Math. 1997, 219, 25-35. (translation of Tr. Mat. Inst. Steklova 1997, 219, 32-43. (in Russian))

[4] Balgimbayeva S.A., Smirnov T.I. Estimates of The Fourier Widths of the Classes Of Periodic Functions With Given Majorant of the Mixed Modulus of Smoothness. Sib. Math. J. 2018, 59 (2), 217-230. doi:10.1134/S0037446618020040 (translation of Sibirsk. Mat. Zh. 2018, 59 (2), 277-292. doi:10.17377/smzh.2018.59.204 (in Russian))

[5] Bari N.K., Stechkin S.B. The best approximations and differential properties of two conjugate functions. Trans. Moscow Math. Soc. 1956, 5, 483-522. (in Russian)

[6] Bazarkhanov D.B. Estimates of the Fourier widths of classes of Nikol'skii-Besov and Lizorkin-Triebel types of periodic functions of several variables. Math. Notes 2010, 87 (1-2), 281-284. doi:10.1134/S0001434610010359 (translation of Mat. Zametki 2010, 87 (2), 305-308. doi:10.4213/mzm8592 (in Russian))

[7] Belinsky E.S. Estimates of entropy numbers and Gaussian measures for classes of functions with bounded mixed derivative. J. Approx. Theory 1998, 93, 114-127. doi:10.1006/jath.1997.3157

[8] Bernstein S.N. Collected work. Vol. II. Constructive theory of functions (1931-1953). Nauka, Moscow, 1954. (in Russian) 
[9] Dũng D. Approximation by trigonometric polynomials of functions of several variables on the torus. Sb. Math. 1988, 59 (1), 247-267. doi:10.1070/SM1988v059n01ABEH003134 (translation of Mat. Sb. 1986, 131(173) (2), $251-271$. (in Russian))

[10] Dũng D., Temlyakov V.N., Ullrich T. Hyperbolic Cross Approximation. Birkhauser, Basel, 2018.

[11] Fedunyk O.V. Estimates of approximation characteristics of the classes $B_{p, \theta}^{\Omega}$ of periodic functions of several variables in the space $L_{q}$. Approx. Theory of Functions and Related Problems: Proc. Inst. Math. NAS Ukr. 2005, 2 (2), 268-294. (in Ukrainian)

[12] Fedunyk-Yaremchuk O.V., Hembars'ka S.B. Estimates of approximative characteristics of the classes $B_{p, \theta}^{\Omega}$ of periodic functions of several variables with given majorant of mixed moduli of continuity in the space $L_{q}$. Carpathian Math. Publ. 2019, 11 (2), 281-295. doi:10.15330/cmp.11.2.281-295

[13] Galeev E.M. Orders of the orthoprojection widths of classes of periodic functions of one and of several variables. Math. Notes 1988, 43 (2), 110-118. doi:10.1007/BF01152547 (translation of Mat. Zametki 1988, 43 (2), $197-211$. (in Russian))

[14] Hembars'kyi M.V., Hembars'ka S.B. Approximate characteristics of the classes $B_{p, \theta}^{\Omega}$ of periodic functions of one variable and many ones. J. Math. Sci. (N.Y.) 2019, 242 (6), 820-832. doi:10.1007/s10958-019-04518-0 (translation of Ukr. Mat. Visn. 2019, 16 (1), 88-104. (in Ukrainian))

[15] Hembarskyi M.V., Hembarska S.B., Solich K.V. The best approximations and widths of the classes of periodic functions of one and several variables in the space $B_{\infty, 1}$. Mat. Stud. 2019, 51 (1), 74-85. doi:10.15330/ms.51.1.74-85 (in Ukrainian)

[16] Lizorkin P.I., Nikol'skii S.M. Spaces of functions with mixed smoothness from the decomposition point of view. Proc. Steklov Inst. Math. 1990, 187, 163-184. (translation of Tr. Mat. Inst. Steklova 1989, 187, 143-161. (in Russian))

[17] Marcinkiewicz J. Quelques remarques Quelques remarques sur l'interpolation. Acta Sci. Math. (Szeged), 1937, 8 (2-3), 127-130. (in French)

[18] Nikol'skii S.M. Functions with dominant mixed derivative, satisfying a multiple Holder condition. Sibirsk. Mat. Zh. 1963, 4 (6) 1342-1364. (in Russian)

[19] Pustovoitov N.N. Representation and approximation of periodic functions of several variables with given mixed modulus of continuity. Anal. Math. 1994, 20, 35-48. doi:10.1007/BF01908917 (in Russian)

[20] Pustovoitov N.N. The orthowidths of classes of multidimensional periodic functions, for which the majorant of mixed continuity moduli contains power and logarithmic multipliers. Anal. Math. 2008, 34, 187-224. doi:10.1007/s10476008-0303-6 (in Russian)

[21] Romanyuk A.S. Approximability of the classes $B_{p, \theta}^{r}$ of periodic functions of several variables by linear methods and best approximations. Sb. Math. 2004, 195 (2), 237-261. doi:10.1070/SM2004v195n02ABEH000801 (translation of Mat. Sb. 2004, 195 (2), 91-116. doi:10.4213/sm801 (in Russian))

[22] Romanyuk A.S. Approximation characteristics and properties of operators of the best approximation of classes of functions from Sobolev and Nikol'skii-Besov spaces. Ukr. Mat. Visn. 2020, 17 (3), 372-395. (in Ukrainian)

[23] Romanyuk A.S. Approximative Characteristics of the Classes of Periodic Functions of Many Variables. Proc. of the Institute of Mathematics of the NAS of Ukraine, Kiev, 2012, 93. (in Russian)

[24] Romanyuk A.S. Diameters and best approximation of the classes $B_{p, \theta}^{r}$ of periodic functions of several variables. Anal. Math. 2011, 37, 181-213. doi:10.1007/s10476-011-0303-9 (in Russian)

[25] Romanyuk A.S. Estimates for Approximation Characteristics of the Besov Classes $B_{p, \theta}^{r}$ of Periodic Functions of Many Variables in the Space $L_{q}$. I. Ukrain. Math. J. 2001, 53 (9), 1473-1482. doi:10.1023/A:1014314708184 (translation of Ukrain. Mat. Zh. 2001, 53 (9), 1224-1231. (in Russian))

[26] Romanyuk A.S. Estimates for Approximation Characteristics of the Besov Classes $B_{p, \theta}^{r}$ of Periodic Functions of Many Variables in the Space $L_{q}$. II. Ukrain. Math. J. 2001, 53 (10), 1703-1711. doi:10.1023/ A:1015200128349 (translation of Ukrain. Mat. Zh. 2001, 53 (10), 1402-1408. (in Russian)) 
[27] Romanyuk A.S., Romanyuk V.S. Approximating characteristics of the classes of periodic multivariate functions in the space $B_{\infty, 1}$. Ukrain. Math. J. 2019, 71 (2), 308-321. doi: 10.1007/s11253-019-01646-3 (translation of Ukrain. Mat. Zh. 2019, 71 (2), 271-282. (in Ukrainian))

[28] Romanyuk A.S., Romanyuk V.S. Estimation of Some Approximating Characteristics of the Classes of Periodic Functions of One and Many Variables. Ukrain. Math. J. 2020, 71 (8), 1257-1272. doi:10.1007/s11253-019-01711-x (translation of Ukrain. Mat. Zh. 2019, 71 (8), 1102-1115. (in Ukrainian))

[29] Stasyuk S.A. Approximation of the Classes $B_{p, \theta}^{\Omega}$ of Periodic Functions of Many Variables in Uniform Metric. Ukrain. Math. J. 2002, 54 (11), 1885-1896. doi:10.1023/A:1024000709997 (translation of Ukrain. Mat. Zh. 2002, 54 (11), 1551-1554. (in Ukrainian))

[30] Stasyuk S.A., Fedunyk O.V. Approximation characteristics of the classes $B_{p, \theta}^{\Omega}$ of periodic functions of many variables. Ukrain. Math. J. 2006, 58 (5), 779-793. doi:10.1007/s11253-006-0101-x (translation of Ukrain. Mat. Zh. 2006, 58 (5), 692-704. (in Ukrainian))

[31] Stechkin S.B. On the order of the best approximations of continuous functions. Izv. Ross. Akad. Nauk Ser. Mat. 1951, 15 (3) 219-242. (in Russian)

[32] Temlyakov V.N. Approximation of functions with bounded mixed derivative. Proc. Steklov Inst. Math. 1989, 178, 1-121. (translation of Tr. Mat. Inst. Steklova 1986, 178, 3-113. (in Russian))

[33] Temlyakov V.N. Approximation of Periodic Functions. Nova Science Publishers, Inc., New York, 1993.

[34] Temlyakov V.N. Approximation of periodic functions of several variables by trigonometric polynomials, and widths of some classes of functions. Izv. Math. 1986, 27 (2), 285-322. doi:10.1070/IM1986v027n02ABEH001179 (translation of Izv. Ross. Akad. Nauk Ser. Mat. 1985, 49 (5), 986-1030. (in Russian))

[35] Temlyakov V.N. Diameters of some classes of functions of several variables. Dokl. Akad. Nauk 1982, 267 (2), $314-$ 317. (in Russian)

[36] Temlyakov V.N. Estimates of the asymptotic characteristics of classes of functions with bounded mixed derivative or difference. Proc. Steklov Inst. Math. 1990, 189, 161-197. (translation of Tr. Mat. Inst. Steklova 1989, 189, $138-168$. (in Russian))

[37] Yongsheng S., Heping W. Representation and approximation of multivariate periodic functions with bounded mixed moduli of smoothness. Tr. Mat. Inst. Steklova 1997, 219, 356-377.

Received 02.10.2020

Федуник-Яремчук О.В., Гембарський М.В., Гембарська С.Б. Апроксимаційні характеристики класів типу Нікольського-Бєсова періодичних функиій у просторі $B_{\infty, 1} / /$ Карпатські матем. публ. — 2020. — T.12, №2. — С. 376-391.

Встановлено точні за порядком оцінки ортопоперечників та близьких до них апроксимаційних характеристик класів типу Нікольського-Бєсова $B_{p, \theta}^{\Omega}$ періодичних функцій однієї та багатьох змінних у просторі $B_{\infty, 1}$. Виявлено, що в багатовимірному випадку $(d \geq 2)$ порядки ортопоперечників згаданих класів функцій реалізуються за наближення їх східчасто-гіперболічними сумами Фур'є, які містять необхідну кількість гармонік. У одновимірному випадку оптимальними, з точки зору порядкових оцінок ортопоперечників відповідних класів функцій, $є$ звичайні частинні суми їх рядів Фур'є. Крім цього слід зазначити, що в одновимірному випадку оцінки розглянутих апроксимаційних характеристик не залежать від параметра $\theta$. Також показано, що норми лінійних операторів, які реалізують порядок найкращого наближення класів $B_{\infty, \theta}^{\Omega}$ у просторі $B_{\infty, 1}$, у багатовимірному випадку є необмеженими.

Ключові слова і фрази: клас типу Нікольського-Бєсова, ортопоперечник, найкраще наближення. 